Petra Storjohann

\title{
Erweiterung von Wortschatzstrukturen durch die Verknüpfung von Synonymen bzw. Antonymen mit kontextgebundenen Kollokationsprofilen
}

\begin{abstract}
Dieser Beitrag zeigt, wie allgemeinsprachige Wörterbücher mit Angaben zur Sinn- und Sachverwandtschaft umgehen sollten, damit sie als geeignetes Hilfsmittel bei der Wortschatzarbeit sowohl im muttersprachlichen als auch im fremdsprachlichen Unterricht eingesetzt werden können. Anhand einiger Beispiele aus dem elexiko-Wörterbuch sollen Möglichkeiten aufgezeigt werden, wie kombinierte lexikalisch-semantische Informationen einen Beitrag zur gezielten Wortschatzerweiterung leisten könnten. Für eine effektive Verankerung sprachlichen und außersprachlichen Wissens sollten Erkenntnisse über das Mentale Lexikon in die Darstellung und Beschreibung von Sprache im Wörterbuch eingebunden werden. Konkrete Vorschläge illustrieren, wie Nachschlagewerke möglicherweise gestaltet werden sollten, um besser als Lehrwerke und Quellen für die Wortschatzarbeit geeignet zu sein. Dafür ist es erforderlich, dass die Dokumentation sprachlicher Zusammenhänge auf unterschiedlichen Ebenen, die angemessene Visualisierung kontextueller Phänomene und explizite Erläuterungen eine entscheidende Rolle spielen.

This paper demonstrates how general monolingual dictionaries should treat information on sense-related lexical items in order to be a useful resource for vocabulary acquisition, applied to native and foreign-language teaching alike. With the help of some examples taken from the reference work elexiko, some feasible options are presented which show how combined lexical-semantic information can specifically contribute to the development of various vocabulary skills. It is argued that insights into the mental lexicon need to be implemented in the representation and description of language in future dictionaries to ensure effective storing of new linguistic and extra-linguistic knowledge. Specific suggestions are made that illustrate what prerequisites reference books need to fulfill in order to be more useful teaching and reference sources for the purpose of systematic vocabulary training. It is therefore necessary, that the documentation of linguistic contexts at different levels, an adequate visualization of contextual phenomena and explicit explanations play a more decisive role.
\end{abstract}

\section{Einleitung}

Sowohl Sprachinteressierte als auch Lernende des Deutschen als Erst-, Zweit- oder Fremdsprache haben ein Interesse an sinn- und sachverwandten Ausdrücken und schlagen in Synonym- bzw. Antonymwörterbüchern sowie in Thesauri nach, um z.B. bei der Textproduktion einen für bestimmte kommunikative Zwecke alternativen Ausdruck zu finden (vgl. z.B. Wiegand 2004, S. 36; Müller 1998, S. 54). Dafür kommen zunehmend elektronische Hilfsmittel in Frage, die in ihren Inhalten oder Präsentationen aber oftmals wenig von traditionellen Nachschlagewerken abweichen. Wörterbücher werden kaum als Materialien für die Wortschatzarbeit im mutter- oder auch im fremdsprachlichen Unterricht eingesetzt, sondern sie dienen als Quelle für schnelle, meist im Privaten stattfindende Nachschlagehandlungen, z.B. beim Verfassen von Texten (siehe Murphy 2013).

Warum werden Nachschlagewerke kaum für systematische Wortschatzarbeit eingesetzt? Viele gängige Synonym- und Antonymwörterbücher sind besonders für Fremdsprachenlernende nur bedingt brauchbar (vgl. Durrell 2001) oder gar, wie es Partington (1998, S. 47) auf den Punkt bringt, ,positively dangerous“. Das hat ganz unterschiedliche Ursachen. 
Bei Internetwörterbüchern liegen z.T. computergenerierte Informationen vor, die nicht redaktionell geprüft und daher nicht hinreichend zuverlässig sind. Bei traditionellen Printwörterbüchern fehlt es dagegen aufgrund von Platzmangel an kontextuellen Erläuterungen der sprachlichen Gebrauchsbedingungen. Oftmals werden in deutschsprachigen Synonymwörterbüchern oder Nachschlagewerken, die Ausdrücke des Bedeutungsgegensatzes beinhalten, lediglich Wortlisten angeboten, die zudem häufig nicht konsequent kontextbezogen zusammengestellt sind (mit Ausnahme des Duden 8). Auf Belege, die wichtige semantische und syntaktische Umgebungen illustrieren, wird meist verzichtet. Das Fehlen umfangreicher kontextuell relevanter Angaben führt dazu, dass Fremdsprachenlernende die angebotenen Informationen nicht genügend semantisieren können, d.h. den lexikalischen Einheiten, auf die sie stoßen, nicht hinreichend kontextuelle Eigenschaften und Restriktionen zuschreiben können. Zusätzlich bieten existierende Synonymiken und Antonymiken oftmals einen geringen Umfang an Einträgen (vgl. Müller 1998). Dies sind nur einige der Gründe, warum Thesauri oder Antonymwörterbücher sowohl im Sprach- als auch im DaF-Unterricht kaum als Lehrmaterial bei der Wortschatzarbeit eingesetzt werden. Dabei könnten Nachschlagewerke dieser Art viel mehr leisten, als bloß ein Repertoire an Wortlisten sinnverwandter Ausdrücke zu sein. Konzeptuell ausgefeilte Angaben zur Sinn- und Sachverwandtschaft können im Unterricht einen gezielten Beitrag zur Wortschatzerweiterung leisten. In diesem Beitrag wird der Frage nachgegangen, welche Angaben für das effektive Erweitern von Wortschatzstrukturen in Hinblick auf sinnverwandte Wörter erforderlich sind, und wie diese präsentiert werden könnten, um Wörterbücher als Lehrwerke und Quellen besser nutzbar zu machen.

\section{Wortschatzarbeit im L1- und L2-Unterricht}

Die Vermittlung des Wortschatzes, also der Wörter, der Strukturen und Regeln ihres Gebrauchs, erhält gegenwärtig im muttersprachlichen Unterricht zu wenig Aufmerksamkeit (z.B. Hecht 2013). Dabei spielt dieses Wissen eine enorme Rolle für die vier kommunikativen Fähigkeiten: Lesen, Schreiben, Hören-Verstehen und Sprechen. Diese gilt es gezielt zu fördern und auszubauen. Die Rolle des Wortschatzes für die sprachliche Entwicklung rückte in den vergangenen Jahren bei Linguisten und Linguistinnen, aber auch bei Psychologen und Psychologinnen in den Vordergrund. Ihre Einschätzung widerspricht der gängigen Schulpraxis sowohl im In- als auch im Ausland (vgl. Milton 2013). In den verschiedenen Lehrplänen wird deutlich, dass die Rolle systematischer und kontinuierlicher Wortschatzarbeit unterschätzt wird (z.B. Späth 2008; Hecht 2013).

Current methods and approaches to language teaching fail to consider how vocabulary should be systematically built into the curriculum or suggest that this would not be appropriate assuming that the acquisition of vocabulary is merely incidental to the process of language learning as a whole. (Milton 2013, S. 73)

Im Gegensatz zu den Fremdsprachen erfolgt im Deutschunterricht eine systematische Wortschatzarbeit jedoch eher selten, da man annimmt, jeder Schüler verfüge über genügend Grundwortschatz und Gelegenheit und Kenntnisse, diesen zu erweitern. (Hecht 2013, S. 2)

Ellis (1997) und Milton (2013) zeigen dagegen, dass Wortschatz für den Erwerb grammatischer Strukturen und für die allgemeine Entwicklung von Sprachhandlungsakten und kommunikativer Kompetenz eine tragende Rolle einnimmt. Daher sollte eine qualitätvolle und durchgängige Sprachbildung im mutter- und zweitsprachlichen Unterricht mehr Gewicht erhalten (vgl. Gogolin et al. 2011; Hecht 2013). 
Im Fremdsprachenunterricht, ungeachtet dessen, ob es z.B. um Englisch im deutschen Sprachraum oder um Deutsch als Fremdsprache geht, hat die Wortschatzarbeit dagegen einen hohen Stellenwert (vgl. Börner 2000). Vokabeln werden als das Grundinventar betrachtet, mit denen sprachliche Äußerungen zusammengestellt werden. Sie bilden die elementaren Bausteine, mit denen Sätze formuliert werden. Dem Üben, Vertiefen und Erweitern von Wortschatz sowie von Wortschatzstrukturen widmet sich die Didaktik des Sprachunterrichts. Methoden des Vokabellernens unterliegen unterschiedlichen Ansätzen. Heutige Lehr- und Lernstrategien zielen darauf ab, sprachliche Zusammenhänge sowie kognitiv gut verankerte lexikalische Strukturen und Beziehungen auch gemeinsam zu vermitteln.

Die gezielte Wortschatzerweiterung spielt eine wesentliche Rolle bei der Entwicklung zahlreicher kognitiver Fähigkeiten und sollte daher generell ein wichtiger Bestandteil des Sprachunterrichts sein, also sowohl für den mutter- und fremdsprachlichen als auch für den fachsprachlichen Spracherwerb. Linguistische Strukturen, wie z.B. Beziehungen der Hyperonymie, Synonymie und des Gegensatzes spielen dabei eine entscheidende Rolle. Sie fördern die Fähigkeit des begrifflichen Denkens und ermöglichen eine ,grobe Ordnung der wahrgenommenen Welt" (Ulrich 2013, S. 315). Synonyme und Antonyme werden bei der Text- und Argumentationsbildung benötigt. Die Beziehung der Über- bzw. Unterordnung wird benötigt, um außersprachliches Wissen zu strukturieren und zu klassifizieren.

[...] Und bei so anspruchsvollen Sprachhandlungen wie dem mündlichen und schriftlichen Argumentieren und Erörtern spielt der sprachliche Ausdruck von Opposition eine entscheidende Rolle, bei der Ordnung der Argumente von Pro und Contra. Die Zusammenstellung vieler Antonyme im Unterricht [...] fördert die Fähigkeit zum begrifflichen Denken und zur präzisen Verbalisierung des Gedachten. [...] Wer das Beziehungsgeflecht von Oberbegriffen, Unterbegriffen und Nebenbegriffen durchschaut, ist in der Lage, die jeweils referentiell betroffenen Teile des Weltwissens systematisch durchzustrukturieren, er kann klassifizieren und definieren. (ebd., S. 315f.)

Auch wenn sich Ulrich (2013) insbesondere auf den Erstspracherwerb und die Vertiefung des Wortschatzes bei Kindern in der Primärsprache bezieht, ist gerade die Kenntnis von Sinnrelationen, sprich von sinn- und sachverwandten Ausdrücken, für den Fremdspracherwerb ebenso wichtig für eine erfolgreiche Kommunikation (vgl. Nation 2001).

\subsection{Wörter und Semantisierungstechniken}

Das Verständnis für Prozesse der Semantisierung ist in der Sprachdidaktik auf die Kognitionswissenschaften zurückzuführen. Man versteht darunter weniger die Bedeutungsvermittlung, als vielmehr die Strategie, Erlerntes zu verarbeiten und sprachliches Wissen im mentalen Lexikon zu verankern. Die Kognitionsforschung belegt klar, dass Wörter im mentalen Lexikon nicht isoliert gespeichert sind, sondern im kommunikativen Kontext mit sprachlichem wie außersprachlichem Wissen ausgestattet und in verschiedenen strukturierten „Wortnetzen" mit untereinander verbundenen Knoten geordnet sind (Spalek 2012, S. 57ff.). Diese Wortnetze sind z.B. themenspezifischer Art, aber auch nach linguistischen Kategorien geordnet (vgl. z.B. Hecht 2013, S. 5). Vernetztes Wortschatzlernen geht auf Forschungsergebnisse zurück, die ergeben haben, dass mehr als 70\% unseres Vokabulars in größeren Einheiten, formelhaften Wendungen (sogenannten Chunks) gespeichert ist (Huber 2001, S. 10). Denn woher wissen Sprecher bzw. Sprecherinnen beispielsweise, in welcher Situation das Wort krank ein angemesseneres Gegenwort zu normal ist als z.B. abartig, ungewöhnlich oder verrückt? Wörter werden kognitiv mit unterschiedlichen Informationen verbunden, und somit können sie gezielt, flexibel und nach kommunikativen Bedürfnissen eingesetzt wer- 
den (vgl. Reinisch 2013). Dafür werden unterschiedliche netzwerkähnliche Verbindungen und das darin abgespeicherte sprachliche und außersprachliche Wissen aktiviert. Die Komplexität solcher mentalen Netze lässt sich nur vereinfacht darstellen (siehe Abb. $1^{1}$ ).

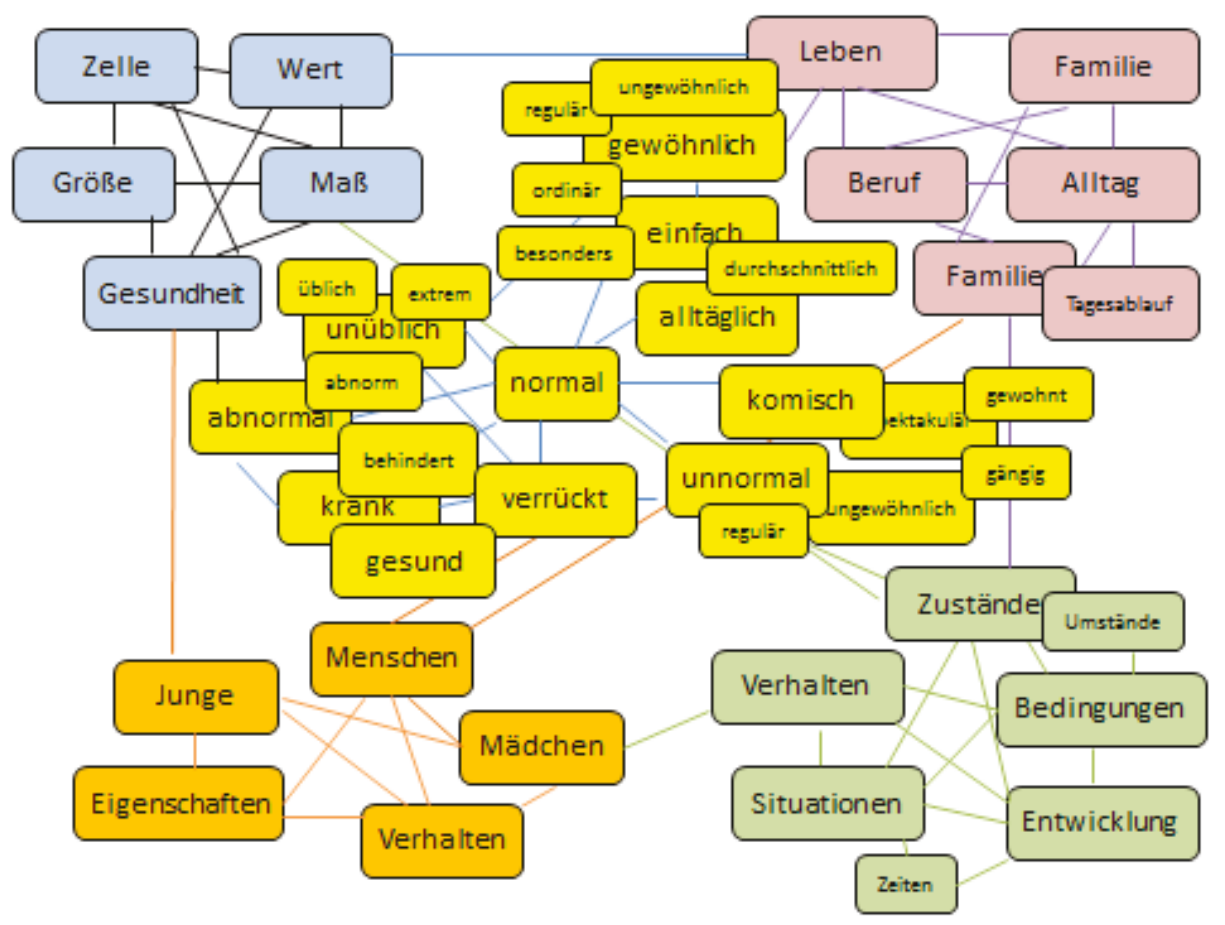

Abb. 1: Vereinfachte Netzstruktur von normal

Psycholog/innen messen der Kognition einen hohen Stellenwert bei der Wortschatzvermittlung bei, denn das mentale Lexikon wird als ,grundlegende Komponente der menschlichen Sprachfähigkeit" verstanden (Köster 2008, S. 887). Die kognitiv abgespeicherten mentalen Netze sind allerdings weitaus flexibler und multimodaler, als mithilfe von Abbildung 1 gezeigt werden kann (vgl. Katsaounis 2006). Das bedeutet, dass der Wortschatzerwerb als eine „,in hohem Grad konstruktive Operation“ (Ulrich 2013, S. 309) aufgefasst werden muss. Interlexematische Verbindungen lassen sich nach Köster (2008) in Begriffsnetze, Wortnetze, syntagmatische Netze, Wortfamilien-, Klang- und Sachnetze unterscheiden. Das Wissen um die vernetzte Verankerung von lexikalischen Strukturen versucht sich auch die Didaktik der Wortschatzarbeit zunutze zu machen (Neveling 2004). Eine „tiefe“ Verarbeitung von Wortschatz wird gewährleistet, wenn Wörter mit anderen Ausdrücken und Erfahrungen verknüpft werden. Um einen höheren Grad an gesicherter Abspeicherung von neuen Wortschatzelementen zu erreichen, wird ein guter Semantisierungsprozess benötigt. Untersuchungen zu Fragen der Semantisierungsprozesse kommen zu sehr ähnlichen Ergebnissen, die nachfolgend kurz skizziert werden.

Eine erfolgreiche Semantisierung kann durch verschiedene Techniken gewährleistet werden, zu denen nach Нaß (2006) u.a. zählen:

- Lernen im Kontext - durch das Verknüpfen mit Weltwissen,

- Definitionen oder Erläuterungen mithilfe von Unter- und Oberbegriffen,

- Bilden von Paraphrasen, Analogien, Synonymen und Antonymen.

1 Die Informationen aus Abbildung 1 basieren auf den Daten des elexiko-Wörterbuchartikels normal. 
Es wird deutlich, dass die erfolgreiche Abspeicherung zu erlernender Vokabeln über Assoziationen zu bestimmten sprachlichen Paradigmen, wie z.B. Wort- oder Themenfelder, gefördert werden kann. Sinn- und sachverwandte Ausdrücke wie Hyponyme, Synonyme und Antonyme etc. spielen damit eine zentrale Rolle bei der Didaktik des Fremdsprachenunterrichts, aber auch bei der primärsprachlichen Wortschatzarbeit. Darüber hinaus zählen zu den grundlegenden Typen assoziativer Verbindungen aber auch sprachliche Koordinationen und Kollokationen. Aus linguistischer Perspektive heißt das, dass sowohl mithilfe paradigmatischer als auch syntagmatischer Relationen Ordnungs- und Zuordnungsübungen erfolgen, die gemeinsames Assoziieren herbeiführen und damit den Semantisierungsprozess fördern. Ein Wort zu kennen heißt daher, über verschiedenes Wissen unterschiedlicher sprachlicher Zusammenhänge $\mathrm{zu}$ verfügen. Die Wortschatzarbeit kann durch verschiedene Kombinationstechniken Synergieeffekte erzielen, die der besseren Verankerung von Vokabeln und entsprechender paradigmatischer wie syntagmatischer Kontexte dienen.

Nation (2001, S. 27) beschreibt in Hinblick auf den Fremdspracherwerb, welches konkrete Wissen für den Erwerb von Wörtern nötig ist, um ein Wort zu kennen. Er unterteilt dabei Wissen in die Rubriken Form, Bedeutung und Verwendung und weist den einzelnen Rubriken konkrete Wissensaspekte zu. In Hinblick auf Bedeutung und Verwendung spielen u.a., aber nicht ausschließlich, folgende Kenntnisse eine Rolle:

\begin{tabular}{|l|l|}
\hline $\begin{array}{l}\text { concepts and } \\
\text { referents }\end{array}$ & $\begin{array}{l}\text { What is included in the concept? } \\
\text { What items can the concept refer to? }\end{array}$ \\
\hline associations & $\begin{array}{l}\text { What other words does this make us think of? } \\
\text { What other words could we use instead of this one? }\end{array}$ \\
\hline use & $\begin{array}{l}\text { In what patterns does the word occur? } \\
\text { In what patterns must we use this word? }\end{array}$ \\
\hline collocations & $\begin{array}{l}\text { What words or types of words occur with this one? } \\
\text { What words or types of words must we use with this one? }\end{array}$ \\
\hline
\end{tabular}

Tab. 1: Wissensrubriken und -aspekte nach Nation (2001, S. 27)

Es wird klar, dass Wörter mit einer komplexen Wissensstruktur verbunden sind, die es zu verstehen und zu lernen gilt. Auch bei Nation (2001) wird erneut deutlich, dass paradigmatische wie syntagmatische Parameter die Wissensstruktur „durchziehen“.

Auch Müller (1994) bringt in diesem Zusammenhang fünf Typen assoziativer Verbindungen zwischen Wörtern im Gedächtnis ins Spiel, die z.T. den oben erwähnten Wissensaspekten stark ähneln. Diese könnten gezielt in die Wortschatzarbeit eingebaut werden, um erfolgreich Wörter in ihrem Gebrauch zu erlernen. Diese fünf Typen sind Koordination (z.B. zwischen Wörtern eines semantischen Feldes wie Salz, Pfeffer, Senf), Kollokationen (z.B. Salz streuen), Subordination (z.B. Vogel: Spatz, Ente, Adler, Eule), Synonyme (z.B. berichtigen $=$ korrigieren $)$ und Antonyme (z.B. gut vs. böse). Auch Müller betont, dass bestimmte Beziehungen syntagmatischer und paradigmatischer Art entscheidende bedeutungs- und verwendungskonstituierende Elemente sind und dadurch assoziative Komponenten bei der Wissensvermittlung sein sollten. Mit anderen Worten, lernt man ein neues Wort, so funktioniert das am besten über das gleichzeitige Lernen seiner kontextuellen Umgebung, um zu wissen, wie es verwendet wird. 
Darüber hinaus ist es wichtig, jedes unbekannte Wort nicht nur mit einer Haupt- bzw. Grundbedeutung zu verknüpfen, sondern komplexere Bedeutungsprofile bei Lexemen herauszuarbeiten und nachhaltig im Gedächtnis abzuspeichern. Von einer guten Wortschatzkompetenz kann nur dann ausgegangen werden, wenn viele Wörter mit ihren potenziellen Lesarten (sprich kontextuellen Verwendungen) erlernt werden (vgl. Ulrich 2013). Und dies geschieht nur über die vielfältige Verwendung von Lexemen in unterschiedlichsten Zusammenhängen und Kontexten. Die Dynamik linguistischer Strukturen, auch von Synonymen und Antonymen, ist kontextuell gebunden und erfordert kognitive Fähigkeiten, sprachliches und außersprachliches Wissen. Das Spektrum kontextueller Möglichkeiten spielt für die Konstruktion von Sprachhandlungen daher eine ebenso große Rolle wie die Kenntnis von rein lexikalischen Einheiten. Anhand konkreter Kontexte lassen sich Verwendungsweisen zwischen semantisch ähnlichen Ausdrücken analysieren und wie Partington (1998, S. 47) es formuliert: ,students will inevitably discover differences in use which are not contained in grammars and dictionaries." Genau diese Information zu konkreten Kontexten steht selten (explizit) in Wörterbüchern und ist für erfolgreiche Vokabelwahl und Wortschatzarbeit im Unterricht notwendig.

\subsection{Wortschatz, Sprachgebrauch und Wörterbücher}

In Bezug auf das Erlernen und Erweitern eines L2-Wortschatzes wird heute durchaus die Lernstrategie des Erstspracherwerbs genutzt. Das heißt, dass mehr oder weniger umfangreich authentische Kommunikation als kontextualisierte Sprachvermittlung erfolgt. Zunehmend wird deshalb die Forderung laut, Fremdsprachenunterricht in der Zielsprache zu halten (Ulrich 2013). Isoliertes Vokabeltraining ermöglicht keinen reflexiven Zugang zum Wortschatz und zu den „Lexembeziehungen im lexikalischen Netzwerk“ (Ulrich 2013, S. 310). Um Sprachkompetenz auszubauen, sollte „,das erworbene Wissen in Sprachhandlungspraxis eingebettet werden“ (ebd.). Kontextualisierter Sprachgebrauch muss sich daher auch in Lehr- und Nachschlagewerken niederschlagen. Das gilt auch für Synonymund Antonymwörterbücher. Tatsächlich basieren nicht alle Nachschlagewerke auf empirischen Untersuchungen tatsächlichen Sprachgebrauchs. Das spiegelt sich auch in teilweise unzuverlässigen bis widersprüchlichen Informationen in Nachschlagewerken wider. Wortschatzarbeit erfolgt im Unterricht daher eher mithilfe von (z.T. eigens) zusammengestellten Arbeitsblättern und Zusatzmaterialien mit Übungen zu Wortfamilien, Wortfeldern, Synonymen oder Antonymen (z.B. Apelt 2001; Ulrich 2007). Für elektronische Wörterbücher meint Reinisch (2013, S. 14) z.B., dass sie vor allem für private Zwecke zu Hause empfehlenswert sind. Sie werden als ,für das selbstständige Arbeiten“ geeignet eingestuft. Daraus wird sichtbar, dass das Wörterbuch weniger eine Quelle für geeignete Übungen für Lehrkräfte darstellt oder gar als ungeeignet wahrgenommen wird. Das liegt auch daran, dass deutschsprachige Synonymiken oder Gegensatzwörterbücher i.d.R. nicht nach unterschiedlichen Benutzergruppen oder gar schulischen Niveaustufen aufgebaut sind und nicht immer Beziehungen dokumentieren, wie sie im tatsächlichen Sprachgebrauch vorkommen und etwa in Korpora systematisch beobachtet werden können. Auch die entsprechenden Teilbereiche der Deutschdidaktik, wie Deutsch als Erstsprache/Muttersprache $(\mathrm{DaM})$, Zweitsprache (DaZ) oder Fremdsprache (DaF), haben wenig Einfluss auf die Gestaltung allgemeinsprachiger Nachschlagewerke. Gezielte DaF-Wörterbücher, die methodisch für ihre Zielgruppe aufbereitet (vgl. Abb. 2) sind, gibt es, sie sind aber i.d.R. stark reduziert und das kontextuelle Spektrum der Stichwörter ist auf Hauptbedeutungen beschränkt. 


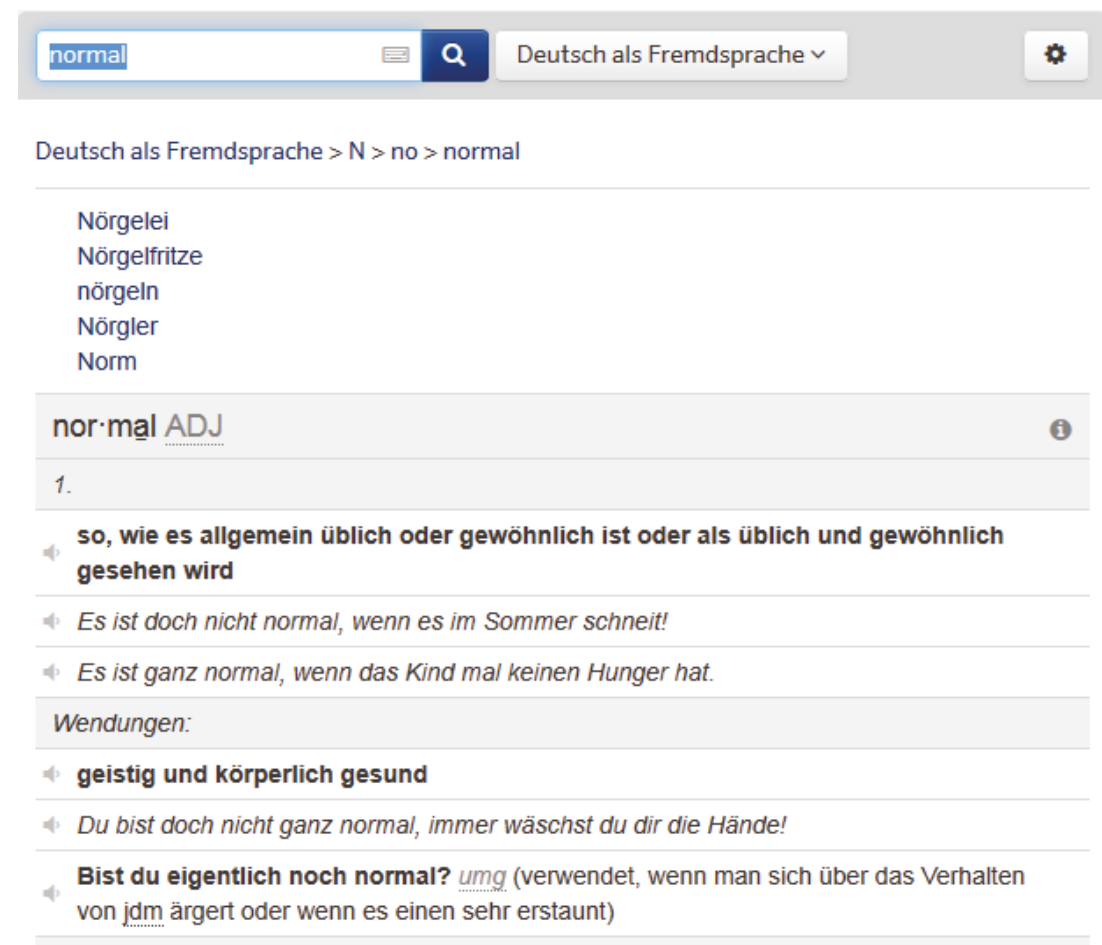

Abb. 2: Eintrag normal im DaF-Bereich des Pons Online-Wörterbuchs²

Der DaF-Bereich des Pons-Wörterbuches richtet sich an Lernende des Deutschen und nicht an Muttersprachler/innen. Synonyme und Antonyme werden hier nicht gesondert genannt, sondern in Paraphrasen eingebettet. Aber das Stichwort normal bietet die gleichen Informationen an, wenn ein Muttersprachler/eine Muttersprachlerin im Bereich „Rechtschreibung“ das Wort nachschlägt. Inwiefern eine Unterscheidung nach Sprachniveau- oder Benutzergruppen überhaupt sinnvoll ist, bleibt umstritten. So argumentiert etwa Nodari (2009), dass die Grenzen zwischen den Lernergruppen nicht klar definiert werden können und daher nur von einer gemeinsamen Deutsch-Fachdidaktik ausgegangen werden sollte, die generell den Menschen und seine kognitiven Fähigkeiten stärker berücksichtigen.

Nach Lernergruppen unterteilte Synonymika und Antonymwörterbücher gibt es wenige und sie sind auf spezifische Ausgangssprachen begrenzt (z.B. für das Englische Durrell 2001). Deutschsprachige Synonym- und Antonymwörterbücher enthalten als Angaben in der Mehrzahl lexikalische Einheiten, die nicht immer im tatsächlichen Sprachgebrauch bedeutungsgleich bzw. kontrastiv verwendet werden, kombiniert mit intuitiv konstruierten Sprachbeispielen. Das liegt u.a. auch an den Methoden und Verfahren, die eingesetzt werden, um sinnverwandte Ausdrücke für ein Stichwort zu erhalten. Nur wenige Wörterbücher nutzen umfangreiche Korpusdaten und korpusgesteuerte Verfahren, um Synonyme/Antonyme zu extrahieren und gleichzeitig ihren tatsächlichen gemeinsamen Gebrauch zu überprüfen (vgl. Storjohann 2012). Derartige Vorgehensweisen sind arbeitsintensiv, bieten aber, im Gegensatz zu automatisch generierten Angaben oder intuitiv erarbeiteten Informationen, eine deutlich höhere Qualität und Zuverlässigkeit. Listen an Synonymen liefern weder relevante Kontexte, noch hinreichende Informationen zu feinen Unterschie-

2 Stichwort normal, siehe http://de.pons.com/\%C3\%BCbersetzung?q=normal\&l=dedx\&in=\&lf=. 
den im Gebrauch. So hält Cruse (2000, S. 390) zu Recht fest: „Most dictionaries are rather bad at discriminating near-synonyms." Um Bezüge zum Gebrauch der Synonyme und Antonyme festhalten zu können, sind aufwendige kontextuelle Untersuchungen der einzelnen potenziellen Wörterbuchkandidaten notwendig. In Hinblick auf englische Synonymwörterbücher fasst z.B. Palmer zusammen:

Dictionaries tell us little about the precise connections between words and their defining synonyms or between the synonyms themselves. (Palmer 1981, S. 91)

Gleiches gilt für die deutschsprachige Lexikografie. Neben der Wahl der Arbeitsverfahren gilt natürlich für gedruckte Wörterbücher, dass die Platzbeschränkung durchaus eine Erklärung dafür ist, dass es keine differenziertere Darstellung gibt. Aber auch zahlreiche elektronische Wörterbücher bieten hier kaum mehr. Nachweislich wird z.B. gern die Thesaurusfunktion von Microsoft Word als populäre Quelle bedeutungsähnlicher Wörter für die schriftliche Textproduktion genutzt (vgl. Murphy 2013). Aber auch hier findet man nur Kandidaten, aber keine Informationen zu den einzelnen Unterschieden zwischen ihnen, um in jeder Situation gezielt das richtige Wort auswählen zu können (vgl. dazu Murphy 2013). Die beschränkte Benutzbarkeit gängiger Nachschlagewerke, (Synonym-, Antonym- und auch Lernerwörterbücher) führt auch zu einem sich allmählich verändernden Nachschlageverhalten.

Whereas fifty years ago, if you wanted to find a synonym, you needed to be near a reference book or a friend with a good vocabulary, these days people wanting to know about word usage may well tweet their question to hundreds of followers, ask it on an internet forum, or email it to a language blogger. Sometimes they do so after encountering 'dictionary-based problems'. (ebd., S. 287)

Die Medien, die zur Wortschatzarbeit im Unterricht eingesetzt werden, haben sich stark verändert (vgl. Götze 2010). Klassische Nachschlagewerke, ob Printwörterbuch oder elektronische Wörterbücher, spielen für die Sprachdidaktik oder Sprachmethodik gegenwärtig keine nennenswerte Rolle. Auf der anderen Seite betont Murphy (2013), dass sich der Zugang zu elektronischen Thesauri und anderen Nachschlagequellen stark vereinfacht und die Nutzung dieser daraufhin auch vermehrt hat. Immer mehr Menschen produzieren aus verschiedensten Gründen Texte. Murphy spricht in diesem Zusammenhang von einer Blütezeit für Lexikograf/innen und der Notwendigkeit, bisherige Thesauren und Synonym-Nachschlagehilfen zu überdenken. Als potenzielles digitales Lehrmaterial und besseres Nachschlagemedium können sie nur genutzt werden, wenn sie sprachdidaktische und sprachmethodologische Aspekte stärker berücksichtigen. Moon (2013) zeigt anhand weniger Beispiele, wie Synonymangaben mit subtilen kontextuellen Informationen über Kollokatoren ergänzt und damit z.B. Lernerwörterbücher und Thesauri zu informativen lehrreichen Quellen werden könnten. Wörterbucheigene Probleme müssen beseitigt werden, indem Erkenntnisse der lexikologischen Forschung besser eingebunden, nötige und umfassende kontextuelle Informationen zur Verfügung gestellt, zuverlässige Angaben angeboten, Informationen des Sprachgebrauchs dokumentiert und Nutzer(innen)bedürfnisse stärker berücksichtigt werden. Und nicht zuletzt spielen auch Fragen der angemessenen Präsentation eine entscheidende Rolle, vor allem für elektronische Medien.

\subsection{Visualisierung von Wortschatzstrukturen}

Die Visualisierung von Informationen, Gedanken und Wissen zählt zu den Möglichkeiten, die eine Verankerung von neuem Wortschatz im mentalen Lexikon unterstützen. Das menschliche Auge kann sehr viele verschiedene optische Details gleichzeitig aufnehmen 
und im Gehirn verarbeiten. Das psychologische Prinzip dahinter ist ein assoziativer Lernprozess, der aufgrund des gemeinsamen visuellen Erfassens Verknüpfungen zwischen verschiedenen, aber durchaus zusammenhängenden Informationen sprachlicher wie außersprachlicher Natur herstellt. Fragen des Designs und der Gestaltung der Zugriffsmöglichkeiten und der Präsentation lexikografischer Angaben sollten in der elektronischen Lexikografie einen hohen Stellenwert haben, da sich zahlreiche neue Optionen der Präsentation und damit der Visualisierung von Wortschatz ergeben. Das eröffnet für Synonym- oder Antonymwörterbücher neue Möglichkeiten.

The shift away from print-based resources means that we can be very imaginative in how synonym information is found and presented. (Murphy 2013, S. 302)

Nur wenige elektronische Wörterbücher sind hinsichtlich ihrer Präsentation empirisch untersucht worden (siehe z.B. Müller-Spitzer 2014). Leider nutzen zahlreiche elektronische Ressourcen längst nicht die Möglichkeiten des digitalen Mediums aus, um Daten innovativ zu präsentieren (vgl. z.B. Tarp 2011). Das gilt auch für die Darstellung von sinnrelationalen Beziehungen wie der Synonymie und Antonymie.

Unterschiedliche Ansätze der Präsentation von Ergebnissen gibt es hier seitens der Computertechnologie und der Lexikografie. Informations- und computertechnologische Visualisierungen bestehen häufig aus automatisch generierten Grafen auf der Basis maschinenlesbarer lexikalisch-semantischer Netze, sogenannter ontologischer Netze, um lexikalische und konzeptuelle Beziehungen zwischen sinn- und sachverwandten Ausdrücken in meist taxonomischer Form darzustellen. Ein solches Wortnetz aus verschiedenen Relationen ist z.B. GermaNet. ${ }^{3}$ Dieses und ähnliche Netze versuchen, die Strukturen des Wortschatzes anhand hierarchischer Grafenstrukturen darzustellen und lexikalische und konzeptuelle Zusammenhänge im Wortschatz zu dokumentieren und damit ein thesaurusähnliches Tool z.B. zum Zwecke automatisierter Disambiguierung zur Verfügung zu stellen. Als Nachschlagewerk für den Sprachunterricht wurden solche Wortnetze nicht konzipiert. ${ }^{4}$ Daneben gibt es automatisierte Visualisierungsverfahren, die relationale Strukturen (auch dynamisch) präsentieren und als Nachschlagewerk angelegt sind. Sie fügen aber kaum nötige Informationen zur Unterscheidung und/oder zum sprachlichen Gebrauch hinzu (siehe Beispiel normal aus www.visuellesynonyme.com in Abb. 3).

3 Zu GermaNet siehe www.sfs.uni-tuebingen.de/GermaNet/.

4 Als grundlegende Zielstellung gibt GermaNet an: „It is primarily intended to serve as a resource for word sense disambiguation which is crucial for natural language applications like information retrieval, the construction of various language technology tools and the annotation of corpora" (www.sfs.unituebingen.de/GermaNet/germanet_structure.shtml). 


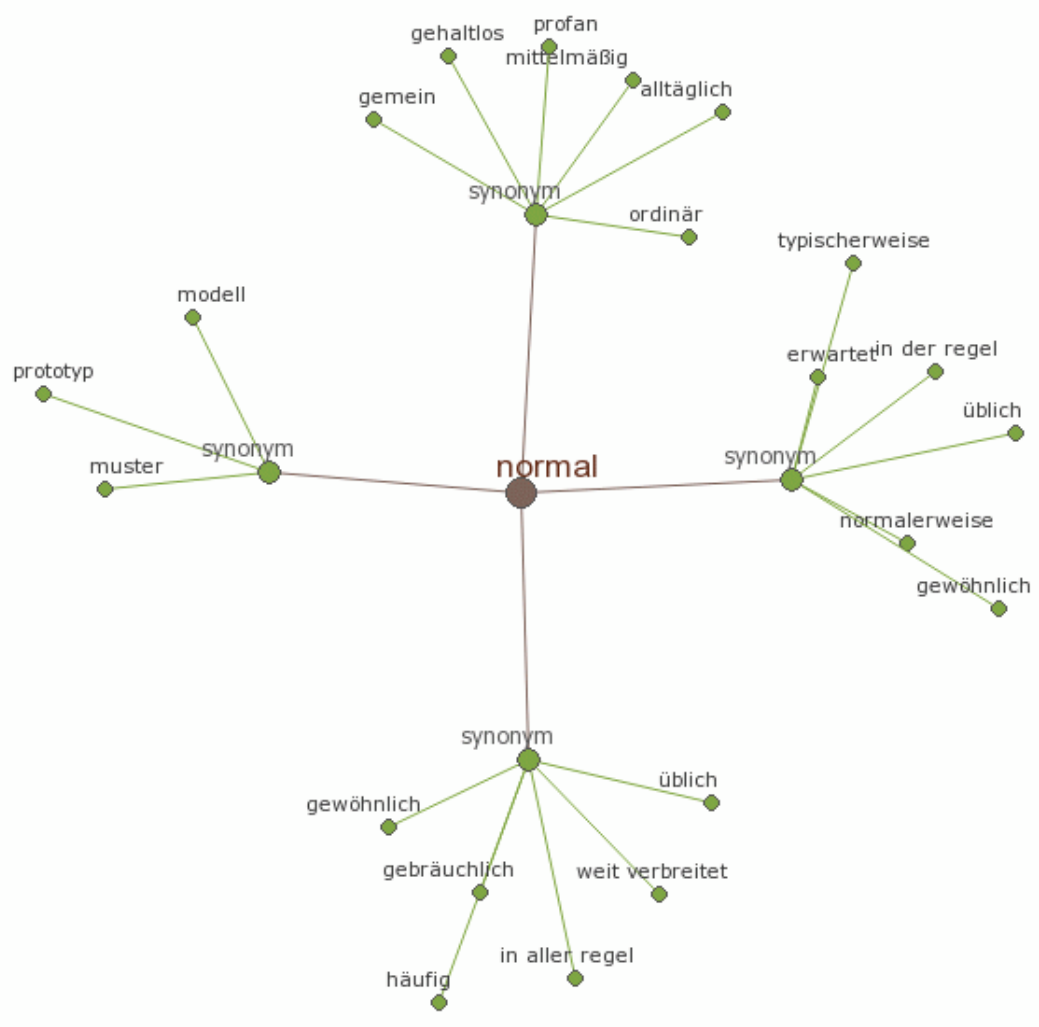

Abb. 3: Visualisierung von Synonymen mittels Software

Es liegen keine Studien oder Berichte darüber vor, ob und wie solche Darstellungen bei der systematischen Wortschatzarbeit bereits eingesetzt werden. Es tut sich aber die Frage auf, welchen Nutzen diese Form der Visualisierung für den Wortschatzerwerb oder die Erweiterung des Vokabulars hat. Die Auflistung der Synonyme geht zwar über eine rein alphabetische Auflistung hinaus, aber die Anordnung der Synonyme in Gruppen ist wenig kontextuell motiviert, daher schlecht nachvollziehbar, und relevante Informationen bezüglich des Gebrauchs fehlen vollständig. Man erhält keinen Mehrwert, da keine sprachlichen Bedingungen zur Unterscheidung bzw. Verwendung aufgeführt werden. Für den englischen Sprachraum sind solche Visualisierungstechniken fortgeschrittener, z.B. beim Visual Thesaurus, der konkrete Kontexte und Verwendungsbeispiele aufnimmt (siehe Abb. 4). Dieser Thesaurus untergliedert die Zuordnung der Synonyme zusätzlich nach syntagmatischen Kriterien, d.h., unmittelbare kontextuelle Umgebungen werden gezeigt, z.B. auch, wie sie in Form von Kollokationen auftreten. Es kommt hier somit zur Verknüpfung von paradigmatischer mit syntagmatischer Information, was die Vermittlung von zusätzlichem Wissen erleichtert. Relevante referenzielle Bezüge werden nach Wortart unterteilt. Nutzer/innen können beispielsweise das englische Adjektiv normal im Zusammenhang mit Substantiven oder mit Adjektiven unterscheiden und anhand konkreter Beschreibungen leichter verschiedene sprachliche und außersprachliche Bezüge herstellen. 


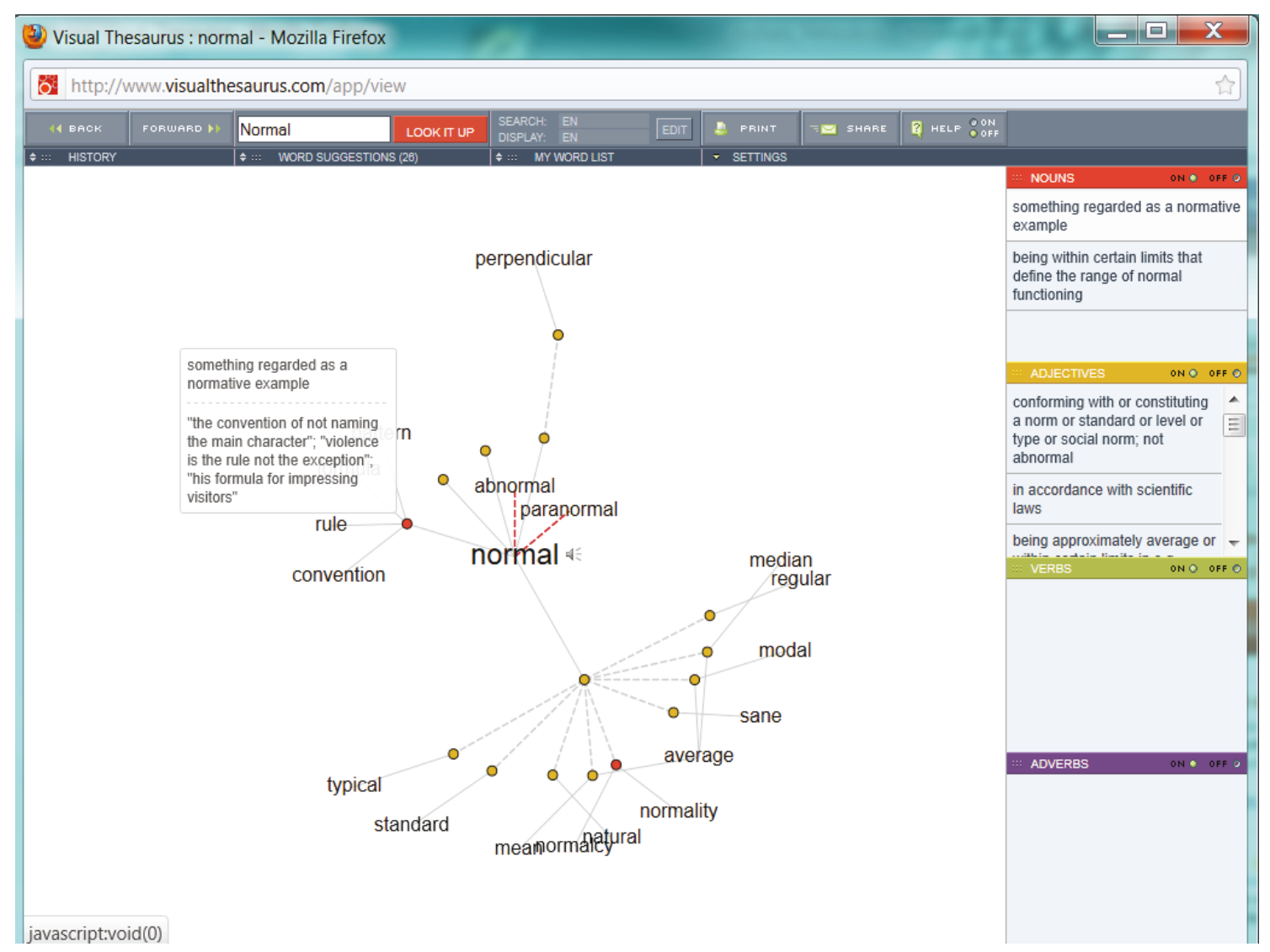

Abb. 4: Visualisierung von englischen Synonymen mit Kontextangaben für normal im Visual Thesaurus

Die (wissenschaftliche) Lexikografie versucht Visualisierungsmöglichkeiten paradigmatischer/syntagmatischer Beziehungen weniger auf der Basis vollautomatisierter Generierung sprachlicher Daten auszuschöpfen, sondern überlegt, wie die Daten, die z.B. korpusgestützt gewonnen, analysiert und linguistisch interpretiert werden, nutzer- und situationsgerecht dargestellt werden können. Sie bemüht sich, den Möglichkeiten des Mediums Internet gerecht zu werden, ohne auf die Zuverlässigkeit der Angaben verzichten zu müssen. Auf gute, richtungsweisende Vorschläge geeigneter Visualisierungen, die linguistisch vertretbar und nutzergerecht angelegt sind, warten Nutzer und Nutzerinnen aber noch.

\section{3. elexiko - und was es bietet}

elexiko ist ein im Aufbau befindliches, umfassendes elektronisches Wörterbuch zur deutschen Gegenwartssprache (vgl. Haß (Hg.) 2005). Es ist das einzige Nachschlagewerk, das seine sinnverwandten Wörter mithilfe unterschiedlicher Analyseverfahren konsequent aus einem Korpus gewinnt, einer kritischen linguistischen Auswertung und Interpretation unterzieht und diese anschließend kontextuell gebunden einordnet, mit einem illustrativen Sprachbeispiel versieht und ggf. auch durch Verwendungshinweise oder lexikografische Kommentare ergänzt (Storjohann 2005a, 2005b, 2006). Die Darstellung lexikalischer Geflechte, die in vielen Wörterbüchern entweder fehlt oder nicht in lexikologisch angemessener Weise erfolgt, war immer ein wesentliches Inhaltsmerkmal von elexiko. Das heißt, dass im Angabebereich „Sinnverwandte Wörter“ nicht nur Synonyme dokumentiert, sondern sämtliche in 
Sinnrelation auftretenden und im Korpus nachweisbaren Beziehungen beschrieben werden. Dazu gehören auch Partero-/Partonyme, Hyper-/Hyponyme und Kohyponyme (inkompatible Partnerwörter) sowie verschiedene Kausal- und Temporalbeziehungen bzw. Relationen der semantischen Steigerung (siehe Hahn et al. 2008). Binäre Verbindungen, wie sie i.d.R. zwischen paradigmatischen Verbindungen vorliegen, werden zudem zusätzlich systematisch geprüft, um fehlende oder fehlerhafte Verknüpfungen zwischen einzelnen Stichwörtern und ihren Partnerwörtern zu vermeiden. D.h., für das Wort normal ist u.a. in der Lesart ,gesund“ das Gegensatzwort krank verzeichnet, und ebenso wird andersherum unter dem Stichwort krank das Antonym normal in der entsprechenden Lesart, nicht gesund' dokumentiert. Die systematische Prüfung aller Beziehungsarten und ggf. deren Korrektur sorgt für ein höchst konsequentes Aufzeichnen binärer Strukturen, das bei zahlreichen anderen Wörterbüchern dieser Art mangelhaft ist (vgl. Paradis/Willners 2007). Dies wird durch die XML-basierte Struktur der Wörterbuchartikel, also seiner inhaltsorientierten Auszeichnung, und mittels zusätzlicher Management-Software realisiert, die computergestützt die Architektur der Artikel systematisch recherchiert und analysiert (siehe dazu Müller-Spitzer 2010; Meyer 2011; Storjohann/Meyer 2012).

Die kontextuellen Umgebungen eines Stichwortes detailliert aufzuschlüsseln, bedeutet für ein Wörterbuch, differenziertere semantische Informationen zum Gebrauch eines Partnerwortes zu dokumentieren. Die lesartengebundene Zuordnung sinnverwandter Wörter und die konkrete Belegung synonymer oder antonymer Verwendungen im Sprachgebrauch erfolgt bei elexiko durch konkrete Belege aus einem Korpus mit tatsächlichen Sprachbeispielen. Abbildung 5 zeigt ausschnitthaft sinnverwandte Ausdrücke für das Suchwort normal in zwei unterschiedlichen Lesarten/Kontexten des Stichwortes.

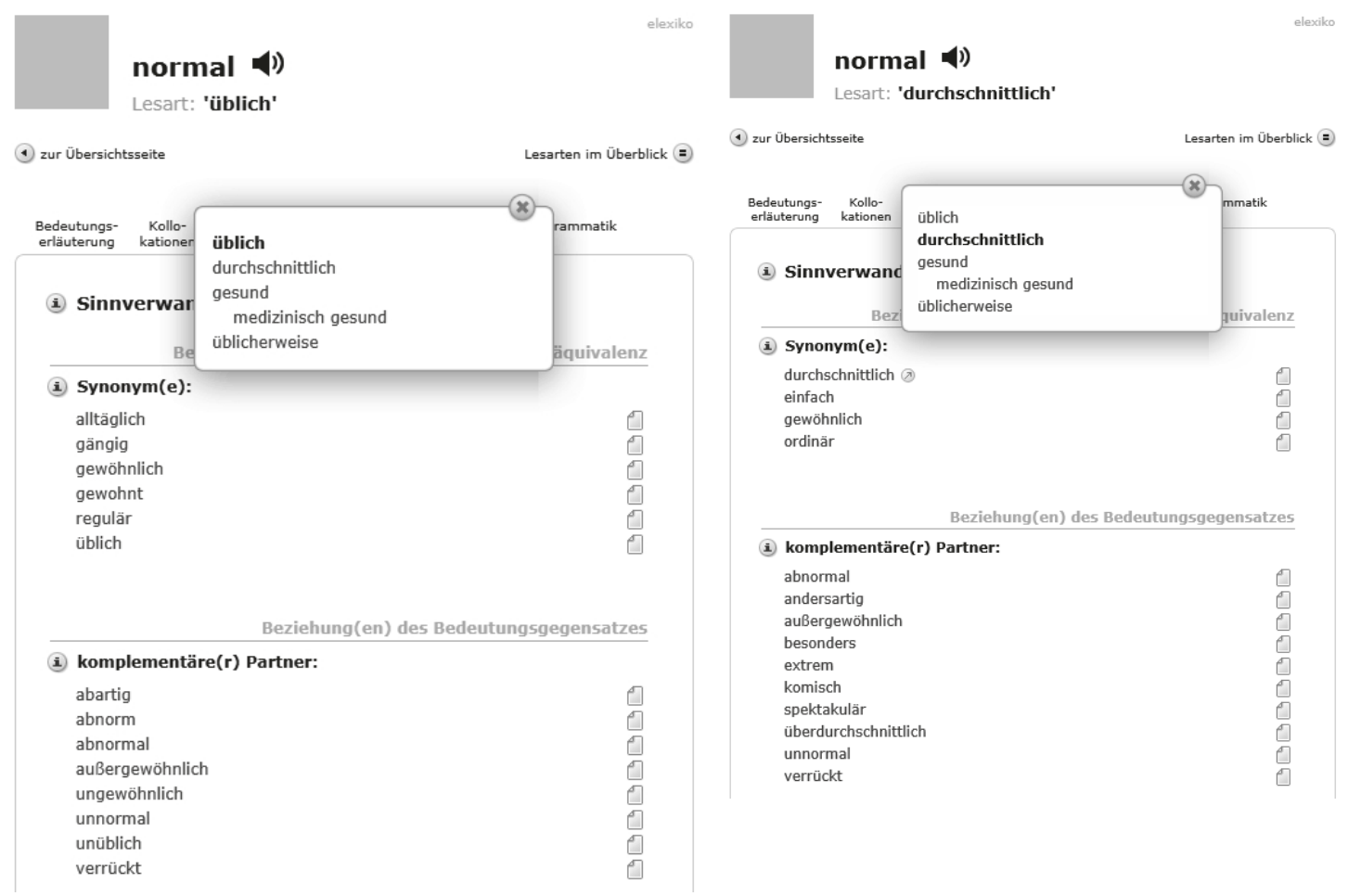

Abb. 5: Lesartengebundene sinnverwandte Wörter für normal in elexiko 
Für das Stichwort normal sind 5 verschiedene Verwendungsweisen in elexiko gebucht. Jede Lesart bzw. jede kontextuelle Sublesart hat ihre zugeordneten Partnerwörter, zu jedem einzelnen Partnerwort erfolgt eine Verlinkung und die Angabe eines authentischen Belegs. Die Aufschlüsselung der sinnrelationalen Partner erfordert eine Disambiguierung polysemer Ausdrücke und eine kontextuelle Interpretation verschiedener Verwendungsweisen. Automatisierte Disambiguierungsverfahren arbeiten hier nicht zuverlässig. ${ }^{5}$ Daher kann diese Interpretation nur durch aufwendige lexikografisch-redaktionelle Verfahren erfolgen. Das Resultat erhöht allerdings die Nutzbarkeit des Wörterbuchs. Die Nutzbarkeit wird außerdem dadurch verbessert, dass Nachschlagende neben der kontextuellen Zuordnung von Synonymen und Antonymen in weiteren Rubriken zusätzliche Informationen zum Kontext erhalten, die die Abgrenzungen der einzelnen Verwendungsweisen verdeutlichen und konkrete Anwendungsbereiche, etwa thematisch-referenzielle Bezüge, illustrieren (z.B. durch Angaben der Bedeutungserläuterung oder der Kollokationen). Das heißt, elexiko ist ein umfangreiches Wörterbuch mit unterschiedlichen Rubriken, die als „Karteikartenreiter" nebeneinander angeordnet sind und die Verwendung und den Gebrauch eines Stichwortes in jeder einzelnen Lesart beschreiben. Aber diese Form der Präsentation hat auch ihren Nachteil. Kontextuell zusammengehörige Informationen zur Verwendung und zum Gebrauch stehen in verschiedenen Rubriken und können nur isoliert rezipiert werden. Nutzer/innen müssen zwischen Wörterbuchrubriken wechseln und können somit nicht immer bestimmte linguistisch-strukturell verbundene Details gemeinsam einsehen. Und auch wenn die Lesart mit ihrer kurzen Etikettierung sichtbar ist, wäre besonders für DaFler die gleichzeitige Darstellung der umfangreicheren Bedeutungserläuterung ebenso wichtig. Auch diese befindet sich bei elexiko in einer separaten Rubrik.

Wie muss also ein Wörterbuch aussehen, das sinn- und sachverwandte Ausdrücke dokumentiert und als Hilfsmittel für die Wortschatzarbeit nutzbar sein soll? Für elexiko liegen empirische Nutzer/innenstudien zu den Inhalten und der Präsentation der Angaben vor (siehe Klosa/Koplenig/Töpel 2014). Die Studien richteten sich an Sprachwissenschaftler/ innen, Übersetzer/innen, Studierende der Sprachwissenschaften, muttersprachliche Deutschlehrer/innen, DaF-Lehrende und nicht-muttersprachliche Deutschlernende. Aus den Befragungen geht u.a. hervor, dass Nutzerinnen und Nutzer die einzelnen Angaberubriken in elexiko als wichtig einschätzen und die zahlreichen Textbelege begrüßen. Nur ca. 37\% der Befragten erwarten bei der Angabe der sinnverwandten Wörter eine grafische Darstellung, die das Stichwort in einem Wortnetz zeigt (ebd., S. 306). Ebenso wurde der Wunsch deutlich, semantische Unterschiede zwischen Synonymen deutlicher zu beschreiben. Die Kombination mit Angaben zu Kollokationen könnte eventuell eine Unterscheidung gewährleisten. Interessant waren auch die Präferenzen bei der Darstellung von Kollokationsstrukturen (ebd., S. 339ff.). Hier bevorzugte die Mehrheit der Befragten andere Präsentationsformen, als die in elexiko vorgegebene Auflistung (alphabetisch untereinander). Die Ergebnisse solcher oder ähnlicher Studien geben Lexikograf/innen wertvolle Einsichten und ermöglichen es ihnen, bestimmte Inhalte und Ansichten künftig benutzerbzw. situationsgerechter zu gestalten.

5 Zu Fragen der Zuverlässigkeit bei automatisch generierten Angaben siehe Storjohann/Schnörch (2014). 


\section{Was elektronische Wörterbücher bieten könnten}

In elexiko kann derzeit die Angabe sinn- und sachverwandter Wörter nur jeweils in Bezug auf eine konkrete Einzelbedeutung betrachtet werden. Auf diese Weise sind die analysierten und mit einem Beleg illustrierten Partnerwörter zwar kontextuell zugeordnet, einen Gesamtüberblick aller Relationspartner über alle Lesarten hinweg bekommt man aber nicht. Als XML-basiertes Wörterbuch, bei dem jede Information granular ausgezeichnet ist, könnte elexiko noch zwei Schritte weiter gehen. Erstens wären Präsentationen denkbar, die die einzelnen Rubriken miteinander vereinen, etwa Angaben zu Kollokationen und zu sinnverwandten Ausdrücken. Zweitens könnten lesartengebundene Ansichten zu einem Spektrum zusammenschmelzen, um das komplexe Verwendungsspektrum eines Ausdrucks auf einen Blick erfassbar zu machen. Dafür ist keine Umstrukturierung der einzelnen Artikelarchitektur nötig, sondern lediglich die darüber liegende HTML-Kodierung muss mittels Transformation der XML-Daten über ein XSLT-Stylesheet verändert werden (siehe Müller-Spitzer 2006). D.h., dass aus lexikografischer Sicht keine inhaltlichen Überarbeitungen oder Ergänzungen nötig sind, es handelt sich lediglich um eine layouttechnische Veränderung.

Zunächst zum ersten Punkt. Die kontextuelle Zuordnung würde zusätzlich nachvollziehbarer gemacht werden, indem Lernenden weitere, kontextuell gebundene linguistische Informationen präsentiert würden, beispielweise durch die Kopplung von sinnverwandten Ausdrücken mit Kollokationsangaben. Partington (1998, S. 29) meint dazu, dass Nutzerinnen und Nutzer einen großen Vorteil davon hätten, Synonymangaben mit Kollokationsangaben zu verbinden, weil sie so die genauen Umstände einsehen können, in denen ein Ausdruck synonym verwendet werden kann, und auf welche unmittelbaren Bezugswörter bzw. Kontexte es sich bezieht.

Comparing the collocational behaviour of so-called synonyms in concordance data may supplement dictionary information and help learners decide in what circumstances substitution of one item for the other is possible in a text. (ebd., S. 29)

Kollokationen sind ein textuelles Phänomen, aber auch eine kognitive Einheit und damit ein psychologisches Phänomen. Das Wissen über Kollokationen gehört zur sprachlichen Kompetenz der Sprecherinnen bzw. Sprecher. Normale Kommunikation hängt stark von Vorhersehbarkeiten vorgefertigter sprachlicher Strukturen, lexikalischer Sequenzen und Muster ab. Sie werden im Gedächtnis bei Zuhörenden evoziert. Die Wahl bestimmter Synonyme oder Antonyme veranlasst daher auch die Entscheidung über dazugehörige Muster, die Muttersprachler/innen im mentalen Lexikon aktivieren können. Diese muss man sich für eine effektive und erfolgreiche Kommunikation aneignen, sowohl in der Muttersprache als auch in der Fremdsprache. Daher ist die Kombination von paradigmatischen mit syntagmatischen Mustern besonders empfehlenswert. Lesartengebundene Kollokationen eines Suchwortes könnten gemeinsam mit ihren Synonymen/Antonymen und auch zusammen mit der Bedeutungserläuterung präsentiert werden, wie u.a. in Abbildung 6 vorgeschlagen wird. 


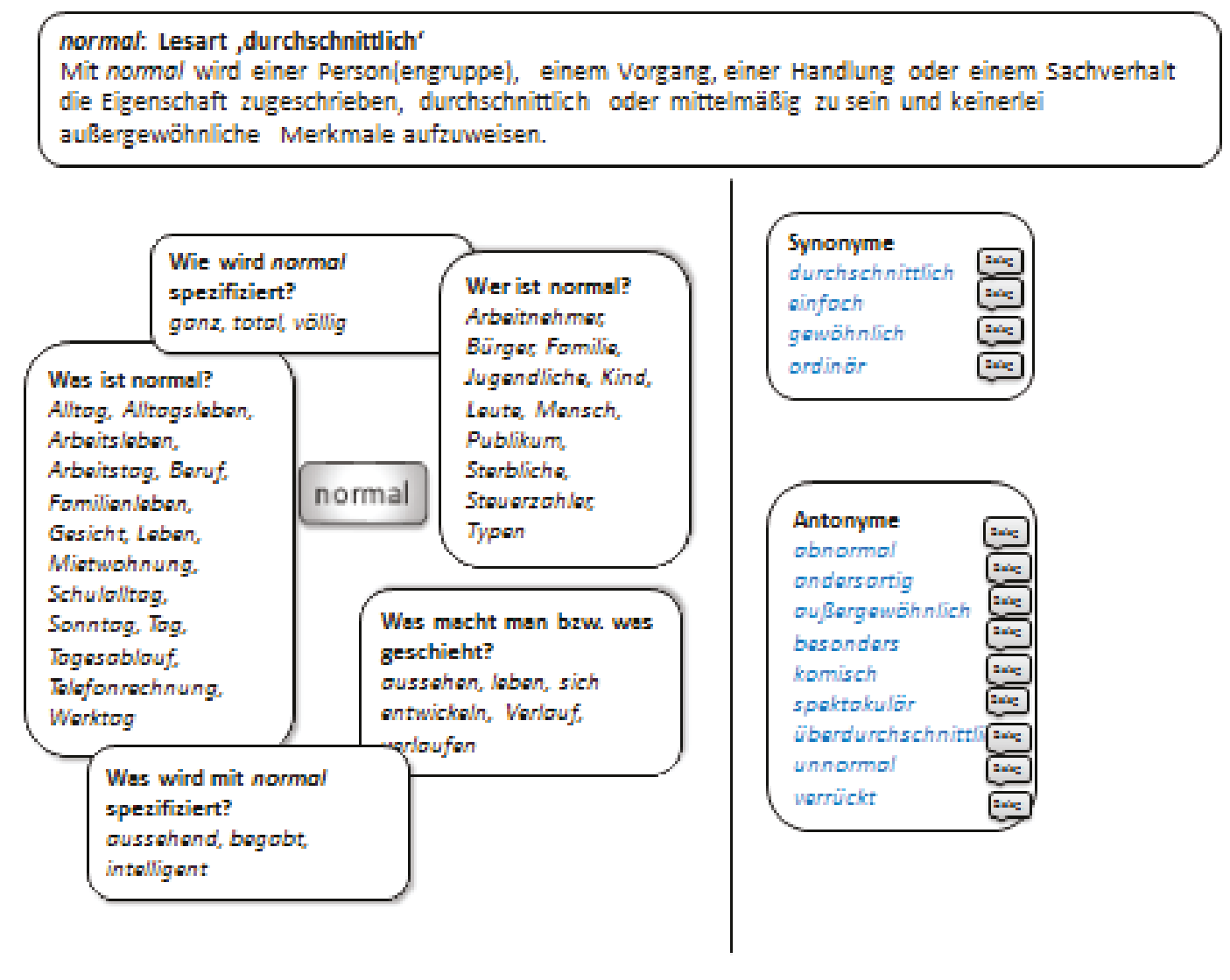

Abb. 6: Vorschlag einer Darstellung der Angaben zu Synonymen/Antonymen mit Kollokatoren und mit der Paraphrase

Eine lesartengebundene Zuordnung und Belegung der Partnerwörter sowie die parallele Auflistung relevanter Kollokatoren, die für die Synonymie- bzw. Gegensatzbeziehung referenziell gebunden sind, sind insbesondere für Fremdsprachenlernende wichtig, um entsprechend kontextuelle Bedingungen erfassen und diese beim Lernen von Synonymen/ Gegensatzwörtern gleichzeitig mit abspeichern zu können. Die begleitenden Belege stützen nicht nur die angegebenen Relationen, sondern lassen sich dadurch auch mit unmittelbaren Kontextwörtern, den Kookkurrenzen, in direkte Beziehung setzen. In der elexikoBenutzerstudie haben sich Befragte bevorzugt für eine Kollokationsangabe entschieden, die die Mitspieler eines Stichwortes um das Stichwort herum gruppiert anzeigt (Klosa/ Koplenig/Töpel 2014, S. 340). Dies wurde hier in der Darstellung berücksichtigt. Derzeit werden Kollokationen in der elexiko-Wörterbuchrubrik „Kollokationen“ untereinander gruppiert und alphabetisch geordnet aufgeführt (siehe dazu Haß (Hg.) 2005).

Zum zweiten Punkt: In Abbildung 6 werden kontextgebundene Informationen zu Kollokatoren mit Angaben zur Sinnverwandtschaft verzahnt, um die Gebrauchsrestriktionen spezieller Synonyme und Antonyme festzuhalten. Dies gilt allerdings nur in Hinblick auf eine spezielle Lesart. Der Ausdruck normal verfügt aber in elexiko über vier weitere Lesarten. Um Synonyme und Antonyme in all ihren Kontexten kontrastiv vergleichen zu können, ließen sich in einem XML-basierten Wörterbuch wie elexiko verschiedene Informationen aus den einzelnen Wörterbuch- bzw. Stichwortrubriken auf eine kompakte Weise darstellen, die einen solchen Vergleich ermöglichen würden. Diese Form der Präsenta- 
tion würde dem Wunsch nachkommen, ein Lexem und seine Sinnverwandtschaft in seinem gesamten Bedeutungsprofil zu dokumentieren. Der Vorteil wäre, dass Sprachinteressierte kontextuelle Zusammenhänge von normal und Einschränkungen anhand der miteinander vernetzten Angaben besser erfassen und diese im Gesamtspektrum einordnen können. Eine Möglichkeit der Darstellung wird in Abbildung 7 vorgestellt.

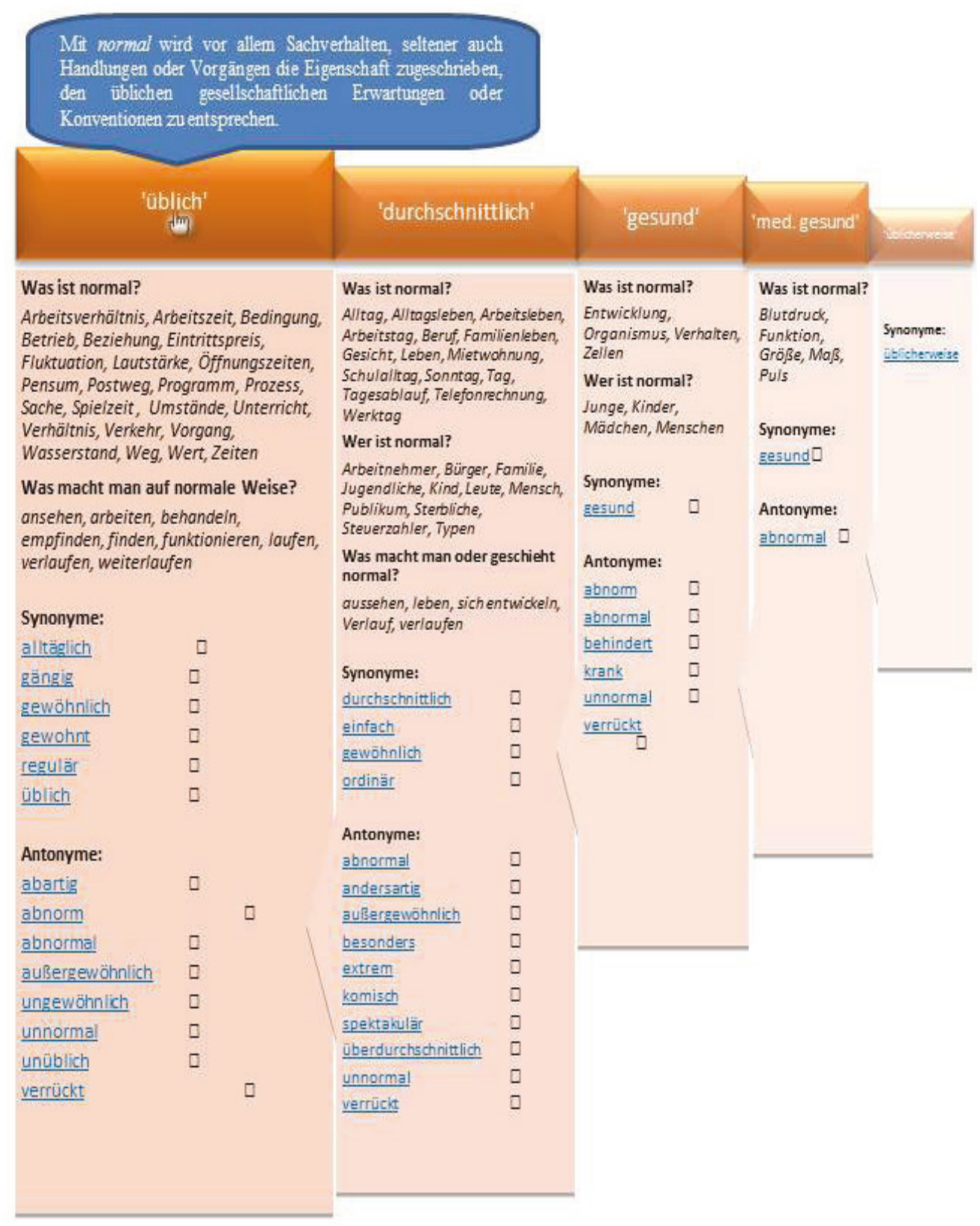

Abb. 7: Mögliche Darstellung aller sinnverwandten Ausdrücke, Kollokatoren und der jeweiligen Paraphrase für ein Stichwort 
Mit dem Ziel der Wortschatzerweiterung erhält man z.B. folgende Informationen auf einen Blick:

1) Der Ausdruck normal hat fünf Einzelbedeutungen.

2) Einige Lesarten verfügen über mehr Inhalte, sind zentraler und typischer als andere, weniger gebräuchliche.

3) In jeder Lesart hat normal verschiedene Synonyme und Antonyme.

4) Manche Synonyme und Antonyme tauchen in mehreren Lesarten auf, andere aber nicht.

5) Welche Antonyme/Synonyme genutzt werden, hängt von referenziellen Bezugswörtern $\mathrm{ab}$.

6) Die Kollokatoren sind nach semantischen Rollen und Funktionen geordnet.

7) Kollokatoren knüpfen Assoziationen zu Themen oder Domänen, in denen normal auftaucht.

8) Die sinnverwandten Wörter werden tatsächlich gebraucht, was durch die Belege illustriert wird.

Die Unterschiede zwischen einzelnen Synonymen lassen sich über unterschiedliche Kollokatoren erschließen. Die Bedeutungserläuterung beinhaltet wichtige referenzielle Aspekte. Diese werden durch Kollokatoren konkret veranschaulicht. Sie dienen damit der beispielhaften Verdeutlichung der Paraphrase und derjenigen Kontexte, die Antonymen oder Synonymen zugewiesen werden. Textbeispiele demonstrieren darüber hinaus syntaktische Besonderheiten des Gebrauchs. Das Stichwort mit seinen Synonymen/Gegensatzwörtern könnte sowohl in seinem Gesamtspektrum an typischen Gebrauchsverwendungen dargestellt und dennoch differenziert lesartengebunden gegliedert werden. Man könnte nicht nur problemlos zwischen den sinnverwandten Wörtern einer Lesart, sondern auch zwischen den unterschiedlichen Verwendungen navigieren. Mit gezielten Ein- und Ausblendfunktionen und interaktiven Modi bestimmter Details könnten Nutzer und Nutzerinnen gelenkt werden. Die einzelnen Lesarten (in diesem Fall horizontal angesiedelte Rubriken) könnten individuell fokussiert werden, indem durch Mausklick die entsprechende Lesart ausgewählt (herausgezoomt) wird, ohne dass die anderen dadurch verschwinden.

Kombinierte Präsentationen können sehr unterschiedlich aussehen und je nach Benutzergruppe speziell definiert werden. Abbildung 7 ist daher nur als eine von vielen Möglichkeiten zu verstehen, die Verschränkungen lexikografischer Informationen auf der Lesartenebene veranschaulicht. So wäre auch eine Darstellung denkbar, die die einzelnen Lesarten untereinander, statt nebeneinander, auflistet. Schwieriger wird die Einbindung aller Verwendungen und der in Abbildung 6 vorgeschlagenen ringartigen Anordnung der Kollokationsgruppen. Denkbar wäre eine vertikale Anordnung, was aber bei hochpolysemen Stichwörtern problematisch ist, weil nicht alles auf einen Blick sichtbar wäre, sondern eine Scrollfunktion zum Einsatz kommen müsste. Für weit stärker polyseme Stichwörter könnte die Darstellung möglicherweise zu komplex werden (z.B. Stichwort frei mit 15 Lesarten). Für diese Stichwörter stellt eine kombinierte Darstellung also eine große Herausforderung dar.

Darstellungen, wie sie in Abbildung 7 vorgeschlagen werden, fehlen bisher vollständig in der deutschsprachigen Lexikografie. Cruse (1986, S. 16) betont: „the meaning of a word is fully reflected in its contextual relations." Eine solche Darstellung wäre deshalb inno- 
vativ, da sie die unterschiedlichen sogenannten syntagmatischen (also Kollokationsstrukturen) und paradigmatischen Beziehungsarten (z.B. Synonymie-/Antonymiebeziehungen) gleichzeitig für sämtliche Verwendungsaspekte des Stichwortes vereint aufzeigt und die Bedeutung des skizzierten Stichwortes bzw. sein semantisches Potenzial in seiner Gesamtheit durchscheinen lässt, ohne bestimmte Details ausblenden zu müssen. Kontextuellstrukturelle Beziehungen eines Stichwortes innerhalb des Wortschatzes lassen sich komplexer und zusammenhängender abbilden, ohne dass auf die Interpretation der Daten verzichtet werden muss, wie dies etwa bei automatisch generierten Wörterbuchangaben der Fall ist. Der Nutzen einer solchen Nachschlageoption wäre demnach immens. Demgegenüber steht allerdings die Erkenntnis aus den Benutzerstudien von elexiko, aus denen hervorging, dass Befragte an die Tradition des gedruckten Wörterbuches gewohnt sind:

Von qualitätvollen neuartigen Angeboten (sei es inhaltlicher Art oder in der Art der Darstellung) müssen die meisten Probanden dagegen wohl noch überzeugt werden (Klosa/Koplenig/Töpel 2014, S. 376).

Laut einer Untersuchung (vgl. Koplenig/Müller-Spitzer 2014, S. 192ff.) wird der sogenannte ,tab view“, also die Karteikartenreiteransicht vor anderen Alternativen, die ebenfalls unterschiedliche Informationen zu einem Stichwort darstellt, bevorzugt. Ansichten, die stark an gedruckte Wörterbücher erinnern, werden dagegen als schlecht bewertet. Die Tatsache, dass in elexiko derzeit manche Informationen erst nach mehrfachem Klicken sichtbar werden, wird als Nachteil dieser Darstellung gesehen. Es soll also so viel Information wie möglich benutzerfreundlich auf einen Blick linguistisch angemessen und zuverlässig präsentiert werden. Auf der einen Seite sind Nachschlagende an bestimmte Formen der traditionellen Präsentation gewöhnt, auf der anderen Seite zeigt sich aber, dass auch alternative Darstellungen angenommen und alternative Darstellungen vom Internet erwartet werden. Das Fazit aber bleibt, dass das gemeinsame Präsentieren unterschiedlicher, aber sprachlich zusammengehöriger, Strukturen im Medium Internet durchaus Optionen bietet, die getestet werden sollten. Ein Wörterbucheintrag, wie er in Abbildung 7 demonstriert wird, bietet für Lehrende und Lernende durchaus Informationen für eigene Lehr- und Übungseinheiten. Kontextgebundene Aufgabenstellungen könnten von Lehrenden leichter selbst erarbeitet werden. Aber auch ohne gezielte Aufgaben können beim Nachschlagen und Stöbern sprachliche und kontextuelle Zusammenhänge visuell besser erfasst werden, die sonst nicht aktiviert bzw. semantisiert werden.

\section{Schlussbemerkungen}

Wörterbücher könnten stärker für die Wortschatzarbeit genutzt werden, um systematisch lexikalische Erweiterungen in Form von Übungen oder Nachschlagehandlungen anzustreben. Dies setzt aber voraus, dass Nachschlagewerke auf der Basis von Korpora erarbeitet, die extrahierten Daten interpretiert und diese für lexikografische Zwecke aufbereitet werden. Es müssen lexikografische Einträge erarbeitet werden, die sprachliche Zusammenhänge auf unterschiedlichen Ebenen aufzeigen, kontextuelle Unterschiede deutlich machen, Sprachbeispiele integrieren, explizite Erläuterungen einbinden und diese Informationen durch gezielte Darstellungen und Visualisierungen intuitiv erfassbar und mental verankerbar machen. Wie Fandrych et al. (2010) zusammenfassen, sollten Einzelphänomene nicht isoliert herausgearbeitet werden, sondern Sprache sollte umfassend in den Blick genommen werden:

Für das Fach DaF/DaZ sind insbesondere linguistische Ansätze und Theorien interessant, die versuchen, Sprache umfassend in den Blick zu nehmen, die funktional orientiert sind, die den Spracherwerb in den 
Blick nehmen und somit Regeln und Regelhaftigkeiten aus der Lernerperspektive beschreiben. Das in den letzten Jahren verstärkt gewachsene Interesse an der Rolle formelhafter Sequenzen (Kollokationen, kommunikative Routinen, idiomatische Prägungen) hat, zusammen mit der in der Spracherwerbsforschung neu bewerteten Rolle von Chunks beim Sprachenlernen, dazu geführt, dass das Verhältnis von Grammatik, Lexikon und kommunikativem Kontext insgesamt neu bewertet wird. (ebd., S. 7)

Ein gut konzipiertes elektronisches Wörterbuch, das verschiedene Aspekte der Wortschatzvermittlung berücksichtigt, könnte ein reiches Informationsangebot für Lehrende und Lernende sein und diese lernpsychologisch unterstützen. Das stellt hohe Ansprüche an Lexikografen bzw. Lexikografinnen sowie an Sprachtechnologinnen und Sprachtechnologen. Aber ebenso stellt es hohe Ansprüche an Lernende beim Umgang mit solchen Ressourcen. Schließlich sollen Nutzer/innen komplexe Strukturen und umfangreiche Informationen zu einem Stichwort, seinen Vernetzungen und seinen Beziehungen kognitiv verankern und nicht den Überblick verlieren. Innovative Präsentationen könnten einen großen Beitrag zur sachgemäßen Erfassung und zur richtigen Methodik des Sichtens und Verankerns von Wortschatz und seinen Strukturen leisten. Damit könnte wortschatzbezogene Arbeit durchgeführt werden, die klug an gebrauchsorientierte und systematische Wortschatzerweiterung herantritt. Wie unterschiedlich erfolgreich innovative Präsentationen wirken, bleibt aber im Detail zu untersuchen.

\section{Literatur}

Apelt, Mary L. (2001): Wortschatz und mehr. Übungen für die Mittel- und Oberstufe. Ismaning: Hueber.

Börner, Wolfgang (2000): Didaktik und Methodik der Wortschatzarbeit: Bestandsaufnahme und Perspektiven. In: Kühn, Peter (Hg.): Wortschatzarbeit in der Diskussion. Hildesheim u.a.: Olms. S. 29-56. (= Studien zu Deutsch als Fremdsprache V).

Cruse, Alan (1986): Lexical Semantics. Cambridge: Cambridge University Press.

Cruse, Alan (2000): Meaning in Language: An Introduction to Semantics and Pragmatics. Oxford: Oxford University Press.

Durrell, Martin (2001): Using German Synonyms. Cambridge: Cambridge University Press.

Ellis, Nick (1997): Vocabulary acquisition: Word structure, collocation, word-class, and meaning. In: Schmitt Norbert/McCarthy, Michael (Hg.), Vocabulary: Description Acquisition and Pedagogy. Cambridge: Cambridge University Press. S. 122-139.

Fandrych, Christian et al. (2010): Deutsch als Fremd- und Zweitsprache als spezifisches Lehr- und Forschungsgebiet. In: Krumm, Hans-Jürgen et al. (Hg.): Deutsch als Fremd- und Zweitsprache. Ein internationales Handbuch, Bd. 1. Berlin/Boston: de Gruyter. S. 1-18.

Gogolin, Ingrid et al. (2011): Durchgängige Sprachbildung. Qualitätsmerkmale für den Unterricht. Münster: Waxmann.

Götze, Lutz (2010): Die Funktion von Medien im Deutsch als Fremd- und Deutsch als Zweitsprache-Unterricht, Deutsch als Fremd- und Zweitsprache. In: Krumm, Hans-Jürgen et al. (Hg.): Deutsch als Fremdund Zweitsprache. Ein internationales Handbuch, Bd. 2. Berlin/Boston: de Gruyter. S. 1199-1263.

Hahn, Marion et al. (2008): elexiko - das elektronische, lexikografisch-lexikologische korpusbasierte Wortschatzinformationssystem. Zur Neukonzeption, Erweiterung und Revision einzelner Angabebereiche. In: Klosa, Annette (Hg.): Lexikografische Portale im Internet. Mannheim: Institut für Deutsche Sprache. S. 57-86. (= OPAL Sonderheft 1/2008).

Haß, Frank (2006): Fachdidaktik Englisch. Stuttgart: Klett.

Haß, Ulrike (Hg.) (2005): Grundfragen der elektronischen Lexikographie. elexiko - das Online-Informationssystem zum deutschen Wortschatz. Berlin/New York: de Gruyter. 
Hecht, Pavla (2013): Wortschatzarbeit im Deutschunterricht der Unterstufe. Norderstedt: Grin-Verlag.

Huber, Konrad (2001): Methodenvorschläge und Hinweise für den Englischunterricht in der 5. und 6. Jahrgangsstufe, Staatsinstitut für Schulpädagogik und Bildungsforschung, München. Online unter: www. realschule.bayern.de/lehrplan/dokumente/e/meth_5u6.pdf. (Stand 5.5.2014)

Katsaounis, Nikolaos (2006): „Funkelnde“ Wörter: zum Entwurf eines multimodalen Wortbedeutungsnetzes als gedächtnisstützende und kognitive Lernstrategie im kommunikativen und medial gestützten Unterricht des Deutschen als Fremdsprache. München: Iudicium.

Klosa, Annette/Koplenig, Alexander/Töpel, Antje (2014): Benutzerwünsche und -meinungen zu dem monolingualen deutschen Onlinewörterbuch elexiko. In: Müller-Spitzer, Carolin (Hg.), S. 281-385.

Koplenig, Alexander/Müller-Spitzer, Carolin (2014): Questions of Design. In: Müller-Spitzer, Carolin (Hg.), S. 189-204.

Köster, Lutz (2008):Wortschatzvermittlung,. In: Helbig, Gerhard (Hg.): Deutsch als Fremdsprache. Ein Internationales Handbuch. Bd. 2. Berlin/New York: de Gruyter. S. 887-893. (= Handbücher zur Sprachund Kommunikationswissenschaft).

Meyer, Peter (2011): vernetziko: A Management Tool for the Lexicographer's Workbench. In: Conference Proceedings of eLEX2011 (Electronic Lexicography in the 21st century: New Applications for New Users), 10_12 November 2011, Bled, Slovenia. Online unter: www.trojina.si/elex2011/Vsebine/ proceedings/eLex2011-25.pdf. (Stand 15.5.2014)

Milton, James (2013): Measuring the contribution of vocabulary knowledge to proficiency in the four skills. In: Bardel, Camilla/Lindqvist, Christina/Laufer, Batia (Hg.): L2 vocabulary acquisition, knowledge and use. Eurosla. S. 57-78. (= Eurosla Monographs Series, 2). Online unter: www.eurosla.org/monographs/ EM02/EM02home.php. (Stand 15.5.2014)

Moon, Rosamund (2013): Braving Synonymy: From Data to Dictionary. In: International Journal of Lexicography 26/3, S. 260-278.

Müller, Bernd-Dietrich (1994): Wortschatzarbeit und Bedeutungsvermittlung. Fernstudieneinheit 8. Fernstudiumprojekt zru Fort- und Weiterbildung im Bereich Germanistik und Deutsch als Fremdsprache. Berlin: Langenscheidt.

Müller, Wolfgang (1998): Möglichkeiten und Perspektiven für die Arbeit mit Synonymiken und Antonymiken. In: Lexicographica 14, S. 54-67.

Müller-Spitzer, Carolin (Hg.) (2014): Using Online Dictionaries. Berlin/Boston: de Gruyter. (= Lexicographica. Series Maior 145).

Müller-Spitzer, Carolin (2006): Das Konzept der Inhaltsstruktur. Ein Ausschnitt aus einer neuen Konzeption für die Modellierung lexikografischer Daten. Mannheim: Institut für Deutsche Sprache. Online unter: www.pub.ids-mannheim.de/laufend/opal/opal06-2.html. (Stand 15.5.2014)

Müller-Spitzer, Carolin (2010): The consistency of sense-related items in dictionaries: Current Status, proposals for modelling and applications in lexicographic practice. In: Storjohann, Petra (Hg.): LexicalSemantic Relations. Theoretical and Practical Perspectives. Amsterdam: Benjamins. S. 145-162.

Murphy, M. Lynne (2013): What we talk about when we talk about synonyms (and what it can tell us about thesauruses). In: International Journal of Lexicography 26/3, S. 279-304.

Nation, Paul I. S. (2001): Learning Vocabulary in Another Language. Cambridge: Cambridge University Press.

Neveling, Christiane (2004): Wörterlernen mit Wörternetzen. Eine Untersuchung zu Wörternetzen als Lernstrategie und als Forschungsverfahren. Tübingen: Narr.

Nodari, Claudio (2009): Deutsch als Fremdsprache/Deutsch als Zweitsprache/Deutsch als Muttersprache Perspektiven Einer Deutschdidaktik. In: Clalüna, Monica/Etterich, Barbara (Hg.): Deutsch unterrichten zwischen DaF, DaZ und DaM. Akten der Zweiten Gesamtschweizerischen Tagung für Deutschlehrerinnen und Deutschlehrer, Bern, 20. und 21. Juni 2008. Sondernummer Rundbrief AkDaF. Stallikon: Käser. S. 11-24. 


\section{Erweiterung von Wortschatzstrukturen}

Palmer, Frank Robert (1981): Semantics. 2. Aufl. Cambridge: Cambridge University Press.

Paradis, Carita/Willners, Caroline (2007): Antonyms in dictionary entries: methodological aspects. In: Linguistica 61/3, S. 261-277.

Partington, Alan (1998): Patterns and Meanings, Series in Corpus Linguistics. Amsterdam/Philadelphia: Benjamins.

Reinisch, Katrin (2013): Wortschatzarbeit im Englischunterricht. In: Sprachsensibler Fachunterricht: Handreichung zur Wortschatzarbeit in den Jahrgangsstufen 5 bis 10. S. 97-124. LISUM, Bildungsserver BerlinBrandenburg. Online unter: www.bildungsserver.berlin-brandenburg.de/publikation_sprachsensibler_ fachunterricht.html. (Stand 15.5.2014)

Spalek, Katharina (2012): Wortproduktion. In: Höhle, Barbara (Hg): Psycholinguistik. 2. Auflage. Berlin: Akademie-Verlag. S. 55-73.

Späth, Christopher (2008): Wortbildung im Deutschunterricht- Wortschatzsarbeit und Sprachreflektion. Norderstedt: Grin-Verlag.

Storjohann, Petra (2005a): Paradigmatische Relationen. In: Haß, Ulrike (Hg.): Grundfragen der elektronischen Lexikographie. elexiko - das Online-Informationssystem zum deutschen Wortschatz. Berlin/New York: de Gruyter. S. 249-264.

Storjohann, Petra (2005b): Corpus-driven vs. corpus-based approach to the study of relational patters. In: Proceedings of the Corpus Linguistics Conference Series, Bd. 1, Nr. 1. in Birmingham. Online unter: www. birmingham.ac.uk/research/activity/corpus/publications/conference-archives/2005-birmingham.aspx. (Stand 5.5.2014)

Storjohann, Petra (2006): Sinnrelationen in Wörterbüchern - Neue Ansätze und Perspektiven. In: EliSe. 2/2005, S. 35-61. Online unter: www.elise.uni-ssen.de/elise02_2005.html. (Stand 15.5.2014)

Storjohann, Petra (2012): Der Einsatz verschiedener Korpusmethoden und -verfahren zur Qualitäts- und Konsistenzsicherung am Beispiel der Ermittlung und Dokumentation von Synonymen und Antonymen. In: Lexicographica 28, S. 121-139.

Storjohann, Petra/Meyer, Peter (2012): Konsistent vernetzte Wörterbucheinträge. In: Zeitschrift für Angewandte Linguistik 56, S. 121-134.

Storjohann, Petra/Schnörch, Ulrich (2014): How can corpus linguistics and dictionary work be profitably combined? In: Bettina Bock (Hg.): Proceedings of 6th ICHLL in Jena 2012. Hamburg: Dr. Kovač. S. 273-284.

Tarp, Sven (2011): Lexicographical and Other e-Tools for Consultation Purposes: Towards the Individualization of Needs satisfaction. In: Bergenholtz, Henning/Fuertes-Olivera, Pedro A. (Hg.): e-Lexicography. The Internet, Digital Initiative and Lexicography. London/New York: Continuum. S. 54-60.

Ulrich, Winfried (2007): Wörter, Wörter, Wörter: Wortschatzarbeit im muttersprachlichen Deutschunterricht: Anleitung und praktische Übungen mit 204 Arbeitsblättern in Form von Kopiervorlagen. Hohengehren: Schneider.

Ulrich, Winfried (2013): Wissenschaftliche Grundlagen der Wortschatzarbeit im Fachunterricht. In: Sprachsensibler Fachunterricht: Handreichung zur Wortschatzarbeit in den Jahrgangsstufen 5 bis 10. S 305-330. LISUM, Bildungsserver Berlin-Brandenburg. Online unter: www.bildungsserver.berlin-brandenburg.de/ publikation_sprachsensibler_fachunterricht.html. (Stand 15.5.2014)

Wiegand, Herbert Ernst (2004): Einführung. In: Dornseiff= Der deutsche Wortschatz nach Sachgruppen. 8., völlig neu bearbeitete und mit einem vollständigen alphabetischen Zugriffsregister versehene Auflage von Uwe Quasthoff. Mit einer lexikographisch-historischen Einführung und einer ausgewählten Bibliographie zur Lexikographie und Onomasiologie von Herbert Ernst Wiegand. Berlin/New York: de Gruyter. S. 9-91. 


\section{Nachschlagewerke und elektronische Ressourcen (Stand 20.5.2014)}

Duden 8: Die sinn- und sachverwandten Wörter. Synonymwörterbuch der deutschen Sprache. Bibliographisches Institut: Mannheim.

elexiko: www.owid.de/wb/elexiko/start.html

GermaNet: www.sfs.uni-tuebingen.de/GermaNet/

Pons Online-Wörterbuch: http://de.pons.com/

Visuelle Synonyme: www.visuellesynonyme.com

Dr. Petra Storjohann

Institut für Deutsche Sprache Mannheim

R5, 6-13

D-68161 Mannheim

E-Mail: storjohann@ids-mannheim.de 